\title{
Effects of Large Dynamic Loads on Power System Stability
}

\author{
M. A. Mahmud ${ }^{\mathrm{a}, *}$, M. J. Hossain ${ }^{\mathrm{b}}$, and H. R. Pota ${ }^{\mathrm{a}}$ \\ ${ }^{a}$ School of Engineering \& Information Technology (SEIT), The University of New South \\ Wales at Australian Defence Force Academy (UNSW@ADFA), Canberra, ACT 2600, \\ Australia \\ ${ }^{b}$ Griffith School of Engineering, Griffith University, Gold Coast, QLD 4222, Australia
}

\begin{abstract}
This paper investigates the critical parameters of power system which affect the stability of the system. The analysis is conducted on both a single machine infinite bus (SMIB) system and a large multi-machine system with dynamic loads. To further investigate the effects of dynamic loads on power systems stability, the effectiveness of conventional as well as modern linear controllers is tested and compared with the variation of loads. The effectiveness is assessed based on the damping of the dominant mode and the analysis in this paper highlights the fact that the dynamic load has substantial effect on the system damping.
\end{abstract}

Keywords: Critical parameters, damping, dynamic loads, linear controllers, power system stability

*Corresponding author: Tel: +61262688199

Email: M.Mahmud@adfa.edu.au (M. A. Mahmud) 


\section{Introduction}

Electric loads play an important role in the analysis of angle and voltage stability of interconnected power systems. Due to the large diverse load components, the changing load composition with time, weather and temperature, and uncertain load characteristic, it is difficult to accurately model the loads for stability studies. The stability of electromechanical oscillations and voltage oscillations between interconnected synchronous generators and loads is necessary for secure system operation because an unsecured system can undergo non-periodic major cascading disturbances, or blackouts, which have serious consequences. Power grids all over the world are experiencing many blackouts in recent years [1] which can be attributed to special causes, such as equipment failure, overload, lightning strokes, or unusual operating conditions.

The secure operation of power systems with the variation of loads has been a challenge for power system engineers since the 1920s [2, 3]. The fundamental phenomenon of secure power system operation is investigated in [4] which has explored a variety of machine loading, machine inertias, and system external impedances with a determination of the oscillation and damping characteristics of voltage or speed following a small disturbance in mechanical torque. Based on this phenomenon, many techniques to assess the stability of the power system have been proposed. In [5], there is an extensive description of power system stabilizers (PSSs) which are now widely used in the power industry. Some improved methodologies of PSS design are proposed in $[6,7]$ which has large disturbance rejection capacity. A Fourier-based sliding method is considered in [8] for secure operation of a power system with large disturbances. Recently, a coordinated PSS design approach is proposed in [9]. In these papers [4]-[9], the power system is mainly considered as a single machine infinite bus (SMIB) system or a multimachine system and linear control techniques are used to ensure the secure operation of the power system. Some nonlinear control techniques are 
also proposed in [10]-[12] for single machine infinite bus (SMIB) system or a multimachine system to obtain a better performance as compared to the traditional linear controllers.

Most of the work as mentioned in the literature [4]-[12], provides an overview of power system stability where the loads are considered as constant impedance loads. Recently, much attention has been paid to the research on the influence of dynamic or static characteristics of loads on power system stability analysis $[13,14]$ and the reasonable representation of loads for different study purpose which is elaborately described in $[15,16]$.

The induction motor loads which are considered as dynamic loads, account for a large portion of electric loads, especially in large industries and air-conditioning in the commercial and residential areas. The induction motors used in system studies are aggregates of a large number of different motors for which detailed data are not directly available; therefore it is important to identify the critical parameters for stability studies. The effects of induction machines on power system stability are focused in [17] where a Hellenic power system is considered for analysis and attention is given to electromechanical oscillations and the critical parameter investigation. In [17] induction motors and synchronous generators are considered separately but practically most of the nonlinearity occurs due to the interconnections between them. Moreover, in [17] some parameters are investigated which affect the stability of the system by neglecting the damping of the system, which is not practical, and finally power system stabilizers are implemented to make the system stable. The critical parameters for a SMIB system as well as large system are also investigated in our previous work [18] by considering all the limitations as presented in [17].

Dynamic stability analysis of power system networks with induction generators has been described in [19] where the induction generators are integrated with wind turbine, i.e., they are not considered as loads. The stability of induction motor networks is nicely described in 
[20] where the induction motors are considered as loads and bifurcation method is used to analyze the stability. In [20], only the slip of the induction motors is considered as dynamic which does not represent all the behaviors of the motors clearly. To analyze the stability of power system with induction motor loads, a conventional PSS which is also called power oscillation damping controller (PODC) is used in [17] and minimax LQG controller is used in $[13,21]$. The minimax LQG controller provides better performance as compared to PODC $[13,21]$. In these papers [15]-[20], the performances of the controllers are tested by applying different types of faults within a certain range of operating points. But in all of these papers, there is no indication about the effectiveness of the controllers with the variation of dynamic loads.

The aim of this paper is to investigate the effects of dynamic loads on the stability of interconnected power systems. Here, the stability of the system with dynamic loads is analyzed by using the concept of critical parameter selection as described from our previous work [18]. In this paper, a PODC designed for power systems with dynamic loads and the effectiveness of the PODC is evaluated with the variation of dynamic loads. Also, the effectiveness of minimax LQG controller, which is referred to as robust PODC (RPODC), is determined with the changes in induction motor loads within the systems. The effectiveness is mainly considered based on the damping of the dominant mode with controller. This paper also addresses the question whether dynamic loads influence the effectiveness of PODC and RPODC with SMIB system and to what extent as well as what is a suitable way of representing induction motor loads for this purpose.

The rest of the paper is organized as follows. In Section 2, the mathematical modeling of an SMIB system with dynamic load is given. Participation factors and eigenvalue analysis which are used to identify the critical parameters is given in Section 3. Section 4 shows the role of critical parameters on the stability of a large system. An overview of PODC and 
RPODC design is presented in Section 5 and Section 6, respectively. The effects of large dynamic loads with PODC and RPODC are shown in Section 7. Finally, the paper is concluded with future trends and further recommendation in Section 8.

\section{Power System Model}

Power systems can be modeled at several different levels of complexity, depending on the intended application of the model. Fig. 1 shows an SMIB system with induction motor loads [22] which is the main focus of this paper as the foundation of this work is built up from this model. Since an SMIB system qualitatively exhibits the important aspects of the behavior of a multimachine power system and is relatively simple to study, it is extremely useful in studying general concepts of power system stability [11].

In this SMIB model, the power is supplied to the load ( $P_{L}=1500 \mathrm{MW}, Q_{L}=150 \mathrm{Mvar}$ ) from the infinite bus and local generator (approximately, ( $P_{G}=300 \mathrm{MW}, Q_{G}=225 \mathrm{Mvar}$ ). The load at bus-2 is made of three parts: (i) a constant impedance load, (ii) an equivalent large induction motor, and (iii) a shunt capacitor for compensation purposes. The major portion of these loads is the equivalent induction motor.

With some typical assumptions, the synchronous generator can be modeled by the following set of differential equations [5]:

$$
\begin{aligned}
& \dot{\delta}=\omega \\
& \dot{\omega}=-\frac{D}{2 H} \omega+\frac{1}{2 H}\left(P_{m}-E_{q}^{\prime} I_{q g}\right) \\
& \dot{E}_{q}^{\prime}=\frac{1}{T_{d o}^{\prime}}\left[E_{f}-\left(X_{d}-X_{d}^{\prime}\right) I_{d g}\right]
\end{aligned}
$$




$$
\dot{V}_{0}=\frac{1}{T_{r}}\left(V_{t}-V_{0}\right)
$$

where $\delta$ is the power angle of the generator, $\omega$ is the rotor speed with respect to synchronous reference, $H$ is the inertia constant of the generator, $P_{m}$ is the mechanical input power to the generator which is assumed to be constant, $D$ is the damping constant of the generator, $E_{q}^{\prime}$ is the quadrature-axis transient voltage, $K_{A}$ is the gain of the exciter amplifier, $T_{d o}^{\prime}$ is the direct-axis open-circuit transient time constant of the generator, $X_{d}$ is the directaxis synchronous reactance, $X_{d}^{\prime}$ is the direct axis transient reactance, $V_{t}=\sqrt{\left(E_{q}^{\prime}-X_{d}^{\prime} I_{d g}\right)^{2}+\left(X_{d}^{\prime} I_{q g}\right)^{2}}$ is the terminal voltage of the generator, $V_{0}$ is the output voltage of the transducer, $T_{r}$ is the time constant of the transducer, $I_{d g}$ and $I_{q g}$ are direct and quadrature axis currents of the generator. The main source of significant nonlinear effects in this model is related to $I_{d g}$ and $I_{q g}$ for which the expressions will be provided at the end of this section.

A simplified transient model of a single cage induction machine is described by the following algebraic-differential equations written in a synchronously-rotating reference frame [23]:

$$
\begin{gathered}
\left(v_{d}+j v_{q}\right)=\left(R_{s}+j X^{\prime}\right)\left(i_{d m}+j i_{q m}\right)+j\left(e_{q m}^{\prime}-j e_{d m}^{\prime}\right) \\
\dot{s}=\frac{1}{2 H_{m}}\left(T_{e}-T_{m}\right) \\
\dot{e}_{q m}^{\prime}=-\frac{1}{T_{d o m}^{\prime}} e_{q m}^{\prime}+\frac{1}{T_{d o m}^{\prime}}\left(X-X^{\prime}\right) i_{d m}-s \omega_{s} e_{d m}^{\prime} \\
\dot{e}_{d m}^{\prime}=-\frac{1}{T_{d o m}^{\prime}} e_{d m}^{\prime}-\frac{1}{T_{d o m}^{\prime}}\left(X-X^{\prime}\right) i_{q m}+s \omega_{s} e_{q m}^{\prime}
\end{gathered}
$$


where $X^{\prime}=X_{s}+\frac{X_{m} X_{r}}{X_{m}+X_{r}}$ is the transient reactance, $R_{s}$ is the stator resistor which is assumed to be zero, $X_{s}$ is the stator reactance, $X_{r}$ is the rotor reactance, $X_{m}$ is the magnetizing reactance, $X=X_{s}+X_{m}$ is the rotor open-circuit reactance, $T_{d o m}^{\prime}$ is the transient open circuit time constant, $T_{m}$ is the torque drawn by the machine, $H_{m}$ is the inertia constant of the motor, $s=1-\omega_{r}$ is the slip of induction motor where $\omega_{r}$ is the relative speed, $T_{e}=e_{d m}^{\prime} i_{d m}+e_{q m}^{\prime} i_{q m}$ is the electrical torque, $e_{d m}^{\prime}$ and $e_{q m}^{\prime}$ are the direct and quadrature axis transient voltages, $i_{d m}$ and $i_{q m}$ are the direct and quadrature axis currents. Here, this model represents the induction machine in it own direct and quadrature axes, which is different from the $\mathrm{d}$ and $\mathrm{q}$ axes of synchronous generator. So axes transformation is used to represent the dynamic elements of both the induction motor and synchronous generator with respect to the same reference frame and to do so we use the following relations:

$$
\begin{gathered}
E_{m}^{\prime}=\sqrt{\left(e_{d m}^{\prime}\right)^{2}+\left(e_{q m}^{\prime}\right)^{2}} \\
\delta_{m}=\tan ^{-1}\left(\frac{-e_{d m}^{\prime}}{e_{q m}^{\prime}}\right) \\
I_{d m}+j I_{q m}=-\left(i_{d m}+j i_{q m}\right) e^{-j \delta_{m}} \\
V_{d}+j V_{q}=-\left(v_{d}+j v_{q}\right) e^{-j \delta_{m}}
\end{gathered}
$$

where the negative sign with $i_{d m}$ and $i_{q m}$ are indicates that they are opposite to $I_{d m}$ and $I_{q m}$ are when expressed in the same reference frame with synchronous generator.

With these relations, a modified third-order induction motor model can be written as follows: 


$$
\begin{gathered}
\left(V_{d}+j V_{q}\right)=-\left(R_{s}+j X^{\prime}\right)\left(I_{d m}+j I_{q m}\right)+j E_{m}^{\prime} \\
\dot{\omega}_{r}=\frac{1}{2 H_{m}}\left(T_{m}-E_{m}^{\prime} I_{q m}\right) \\
\dot{E}_{m}^{\prime}=-\frac{1}{T_{d o m}^{\prime}}\left[E_{m}^{\prime}+\left(X-X^{\prime}\right) I_{d m}\right] \\
\dot{\delta}_{m}=-\omega_{r}-\frac{X-X^{\prime}}{T_{d o m}^{\prime} E_{m}^{\prime}} I_{q m}
\end{gathered}
$$

To complete the model, the equation of $I_{d g}, I_{q g}, I_{d m}$, and $I_{q m}$ can be written as follows:

$$
\begin{gathered}
I_{d g}=-\frac{E_{q}^{\prime}}{X_{d}^{\prime}}+\frac{V_{\mathrm{i} n f}}{X_{d}^{\prime}+X_{T}+X_{e}} \cos \delta+\frac{E_{m}^{\prime}}{X_{d}^{\prime}+X_{T}} \cos \left(\delta_{m}-\delta\right) \\
I_{q g}=\frac{V_{\mathrm{i} n f}}{X_{d}^{\prime}+X_{T}+X_{e}} \sin \delta-\frac{E_{m}^{\prime}}{X_{d}^{\prime}+X_{T}} \sin \left(\delta_{m}-\delta\right) \\
I_{d m}=-\frac{E_{m}^{\prime}}{X^{\prime}}+\frac{V_{\mathrm{i} n f}}{X_{e}} \cos \delta_{m}+\frac{E_{q}^{\prime}}{X_{d}^{\prime}+X_{T}} \cos \left(\delta-\delta_{m}\right) \\
I_{q m}=\frac{V_{\mathrm{i} n f}}{X_{e}} \sin \delta-\frac{E_{q}^{\prime}}{X_{d}^{\prime}+X_{T}} \sin \left(\delta-\delta_{m}\right)
\end{gathered}
$$

where $V_{\mathrm{inf}}$ is the infinite bus voltage, $X_{T}$ is the reactance of the transformer, and $X_{e}$ is the reactance of the transmission lines.

By linearizing equation (1)-(7) we can represent the overall linearized system as:

$$
\begin{aligned}
& \Delta \dot{x}=A \Delta x+B \Delta u \\
& y=C \Delta x
\end{aligned}
$$


where the state vector $\Delta x$ is given by

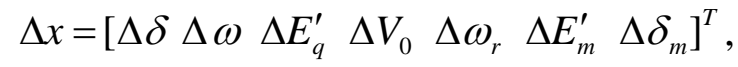

$A$ is the system matrix, $B$ is the input matrix, $C$ is the output matrix, and $y$ is the output vector.

\section{Critical Parameter Analysis}

The linearized system is unstable with one mode in the right-half-plane. The eigenvalues of the SMIB system with dynamic load and participation factors for the unstable mode of the system are shown in Table 1 and Table 2.

Table 1: Eigenvalues without any variation of parameters

\begin{tabular}{|c|}
\hline Eigenvalues \\
\hline$-1.1056 \pm 19.0242 \mathrm{i}$ \\
\hline $0.4689 \pm 13.1049 \mathrm{i}$ \\
\hline$-3.9657 \pm 11.5717 \mathrm{i}$ \\
\hline-3.9686 \\
\hline
\end{tabular}

Table 2: Participation factors without any variation of parameters

\begin{tabular}{|l|c|c|c|c|c|c|c|}
\hline States of the system & $\delta$ & $\omega$ & $E_{q}^{\prime}$ & $V_{0}$ & $\omega_{r}$ & $E_{m}^{\prime}$ & $\delta_{m}$ \\
\hline Participation factor of & 0.4004 & 0.1339 & 0.6519 & 0.06 & 0.31 & 0.6110 & 0.1672 \\
\hline
\end{tabular}




\begin{tabular}{|l|l|l|l|l|l|l|l|}
\hline unstable mode (Mode 2) & & & & & & & \\
\hline
\end{tabular}

From the eigenvalues in Table 1 , it is seen that the system has unstable mode 2 with some low frequency oscillations. The participation factors in Table 2 show that the states $\delta$, $E_{q}^{\prime}$, and $E_{m}^{\prime}$ have the highest participation in this unstable mode which means that there are both angle instability and voltage instability problems in the system. Therefore, now if the elements in the $A$ matrix related to $\delta, E_{q}^{\prime}$, and $E_{m}^{\prime}$ are varied then the system is likely to become stable. The following elements of $A: a_{11}, a_{13}, a_{16}, a_{21}, a_{23}, a_{26}, a_{31}, a_{33}, a_{36}, a_{41}$, $a_{43}, a_{46}, a_{51}, a_{53}, a_{56}, a_{61}, a_{62}, a_{63}, a_{66}, a_{71}, a_{73}$, and $a_{76}$, depend on the system parameters which affect the stability.

By varying the parameters related to the states as mentioned above, it is observed that only the direct axis open circuit time constant of the induction motor $T_{d o m}^{\prime}$, affects the stability of the system when the damping is provided by the synchronous generator. But when no damping is provided by the synchronous generators, the inertia constant of the induction machine also affects the system stability. For nominal value of $T_{d o m}^{\prime}$ equal to 18.7 , the system is unstable. After varying the value of direct axis open circuit time constant of the induction motor, $T_{d o m}^{\prime}$, from 18.7 to 18 or less than 18 , the system becomes stable and the eigenvalues are shown in Table 3.

Table 3: Eigenvalues with the variation of parameter 


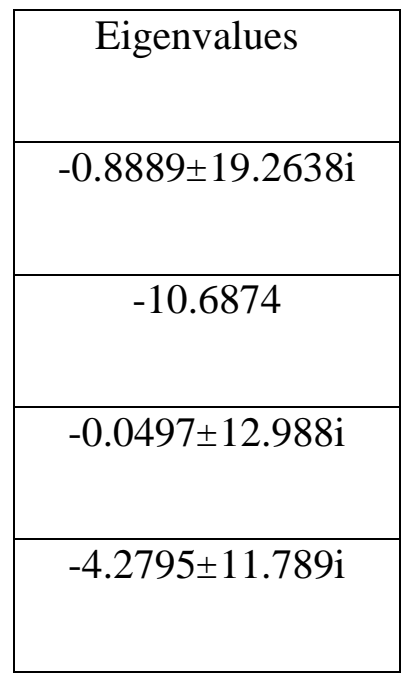

To make the system stable, the value of $T_{d o m}^{\prime}$ is reduced from its nominal value. Though the system is stable, still there are three oscillating modes. These modes are dominated by the voltage as well as angle dynamics. Small variation of the exciter gain $K_{A}$ does not affect the power system stability. But if $K_{A}$ is set to a very high value, the system is stable with high frequency oscillation. On the other hand, very low values of $K_{A}$ make the system unstable.

Fig. 2 shows the variation in the damping of mode 2 with change in $T_{d o m}^{\prime}$. From Fig. 2 , it can be seen that with the small variation of the induction motor direct axis rotor open circuit time constant, the damping changes dramatically. If the $T_{d o m}^{\prime}$ of the induction motor is varied from 17.9 to 18.1 , the damping of mode 2 changes from -0.004 to +0.004 . Fig. 3 describes the damping of mode 2 with variation in the exciter gain, $K_{A}$. From Fig. 3, it is seen that if the exciter gain varies from 25 to 50 , the damping of mode 2 varies from -0.001 to 0.008 . In conclusion, the variation of $T_{d o m}^{\prime}$ is more sensitive to the system stability as compared to the variation of the exciter gain as shown in Fig. 2 and Fig. 3. From Fig. 3, it is 
seen that the system is stable for all values of $T_{d o m}^{\prime} \leq 18$. The role of the critical parameters on a large system is considered in the next section.

\section{Role of Critical Parameters on Large System}

The sensitivity of large power system to the critical parameters is analyzed in this section. For this analysis, a 10-generator, 39-bus New England system shown in Fig. 4 is considered.

The system is unstable and the eigenvalues of dominant unstable mode are $0.35031 \pm 2.8725 \mathrm{i}$. In this mode, the voltage states as well as the angle states of generators at bus-34 and bus-38 have the highest participation in the system instability.

Next, the induction motor loads are connected at bus-4, bus- 8 and bus-20. After connecting these dynamic loads, the system is unstable with dominant eigenvalues $0.31730 \pm 3.3886 \mathrm{i}$. For this mode, the voltage state of generator at bus-38 and the voltage states of induction motor connected at bus-20 have higher participating factor than that of generator at bus-34 and induction motors at other buses.

When the same critical parameter as in the previous section i.e., the direct axis open circuit time constant of the induction motor $T_{d o m}^{\prime}$, is changed, still the system is unstable with eigenvalues $0.29444 \pm 3.4030$ i. Finally, a stable system is obtained by varying $T_{d o m}^{\prime}$ only. At some lower values of $T_{d o m}^{\prime}, 100$ times less than the nominal values, all the eigenvalues of the system are in the left-half complex plane. In this case, the variation of $T_{d o m}^{\prime}$ is more as compared to that of in the previous section. This is due to the large inertia of the system. However, the main concept for critical parameter investigation is true for a large system. 


\section{Overview of PODC Design}

A PODC is designed to damp electromechanical oscillations caused by the large generator inertia and very low damping. The control objective in the PODC design is to increase damping of the electromechanical mode by controlling the synchronous generator excitation systems using an auxiliary signal to the automatic voltage regulator (AVR). Fig. 5 shows the block diagram of excitation system, including the AVR and PODC [5] which is considered in this section.

The dynamics of PODC can be described by the following two equations [5]:

$$
\begin{aligned}
& \dot{v}_{2}=-\frac{1}{T_{w}} v_{2}+K_{S T A B}\left[-\frac{D}{2 H} \omega++\frac{1}{2 H}\left(P_{m}-E_{q}^{\prime} I_{q g}\right)\right] \\
& \dot{v}_{s}=-\frac{1}{T_{2}} v_{s}+\frac{1}{T_{2}} v_{2}+\frac{T_{1}}{T_{2}}\left[-\frac{1}{T_{w}} v_{2}+K_{S T A B}\left(-\frac{D}{2 H} \omega+\frac{1}{2 H}\left(P_{m}-E_{q}^{\prime} I_{q g}\right)\right)\right]
\end{aligned}
$$

where $K_{S T A B}$ is the gain of power oscillation damping controller, $T_{w}$ is the time constant of the washout block, $T_{1}$ and $T_{2}$ are the time constants of the phase compensation block, $v_{2}$ is the output of the washout block and $v_{s}$ is the stabilizing signal which is the output of the phase compensation block.

The PODC is designed in a very similar way to the power system stabilizer (PSS) [5]. The parameters of the designed PODC are as follows:

$$
T_{1}=0.0823 \mathrm{~s}, T_{2}=0.0141 \mathrm{~s}, T_{w}=1 \mathrm{~s} \text {, and } K_{S T A B}=5.7 \text {. }
$$

The eigenvalues of the SMIB system as shown in Fig. 1 are shown in Table 4 when a PODC is applied to the synchronous generator. In this case, the amount of induction motor load is 1000 MW. From Table 4, it is seen that the system is stable which was unstable without a 
PODC as shown in Table 1. This happens due to the supply of additional damping through the PODC.

Table 4: Eigenvalues with PODC

\begin{tabular}{|c|}
\hline Eigenvalues \\
\hline-70.9220 \\
\hline$-0.0076 \pm 46.5101 \mathrm{i}$ \\
\hline$-1.4797 \pm 30.1252 \mathrm{i}$ \\
\hline-19.1221 \\
\hline-1.0305 \\
\hline$-4.3125 \pm 5.4705 \mathrm{i}$ \\
\hline
\end{tabular}

Table 5: Participation factors with PODC when the dynamic load is 1000 MW

\begin{tabular}{|l|c|c|c|c|c|c|c|}
\hline States of the system & $\delta$ & $\omega$ & $E_{q}^{\prime}$ & $V_{0}$ & $\omega_{r}$ & $E_{m}^{\prime}$ & $\delta_{m}$ \\
\hline Participation factor of & 0.013 & 0.0921 & 0.6625 & 0.059 & 0.30 & 0.6210 & 0.1672 \\
unstable mode (Mode 2) & & & & & & & \\
\hline
\end{tabular}


Table 6: Participation factors with PODC when the dynamic load is $1250 \mathrm{MW}$

\begin{tabular}{|l|c|c|c|c|c|c|c|}
\hline States of the system & $\delta$ & $\omega$ & $E_{q}^{\prime}$ & $V_{0}$ & $\omega_{r}$ & $E_{m}^{\prime}$ & $\delta_{m}$ \\
\hline Participation factor of & 0.147 & 0.1281 & 1 & 0.048 & 0.30 & 0.815 & 0.1824 \\
unstable mode (Mode 2) & & & & & & & \\
\hline
\end{tabular}

At this stage, the state participation factor is shown in Table 5 from where it can be seen that the application of PSS reduces the participation of the rotor angle of the synchronous generator. But the voltage mode of the synchronous generator and induction motor is dominating the stability as their participation is more. When the induction motor load is varied from $1000 \mathrm{MW}$ to $1250 \mathrm{MW}$, the participation factors are given in Table 6. From Table 6 , it can be seen that the participation of voltage modes are increasing and still dominating. Thus electrometrical mode is no longer dominating mode for the stability with the variation of dynamic loads.

\section{Overview of RPODC Design}

To design RPODC, a linearization scheme is proposed in [13] which includes the bound of the Cauchy remainder in Taylor series expansion as uncertain term in the controller design. Let $\left(x_{0}, u_{0}\right)$ be an arbitrary point in the control space, using mean-value theorem [24], the system (1)-(7) can be rewritten as follows:

$$
\dot{x}=f\left(x_{0}, u_{0}\right)+L\left(x-x_{0}\right)+M\left(u-u_{0}\right)
$$

where 


$$
\begin{gathered}
L=\left[\left.\left.\frac{\partial f_{1}}{\partial x}\right|_{\substack{x=x^{* 11} \\
u=u^{* 1}}} \cdots \frac{\partial f_{7}}{\partial x}\right|_{\substack{x=x^{* 7} \\
u=u^{* 7}}}\right]^{T} \\
M=\left[\left.\left.\frac{\partial f_{1}}{\partial u}\right|_{\substack{x=x^{* 1} \\
u=u^{* 1}}} \cdots \frac{\partial f_{7}}{\partial u}\right|_{\substack{x=x^{* 7} \\
u=u^{* 7}}}\right]^{T}
\end{gathered}
$$

Here $\left(x^{* p}, u^{* p}\right), p=1, \cdots, 7$ denote points lying on the line segment connecting $(x, u)$ and $\left(x_{0}, u_{0}\right), f=\left[f_{1} \cdots f_{7}\right]^{T}$ denotes the vector function on the right-hand side of the vector differential equation (1)-(7). The nonlinearity of the system (1)-(7) is captured through the nonlinear dependencies $x^{* p}=\Phi_{1 p}\left(x, u, x_{0}, u_{0}\right)$ and $u^{* p}=\Phi_{2 p}\left(x, u, x_{0}, u_{0}\right), p=1, \ldots, 7$.

Letting $\left(x_{0}, u_{0}\right)$ be the equilibrium point about which the trajectory is to be stabilized and defining $\Delta x=x-x_{0}$ and $\Delta u=u-u_{0}$, it is possible to rewrite (12) as follows

$$
\Delta \dot{x}=\dot{x}-\dot{x}_{0}=L\left(x-x_{0}\right)+M\left(u-u_{0}\right)=A \Delta x+B_{1} \Delta u+(L-A) \Delta x
$$

where $A=\left.\frac{\partial f}{\partial x}\right|_{\substack{x=x_{0} \\ u=u_{0}}}$ and $B_{1}=\left.\frac{\partial f}{\partial u}\right|_{\substack{x=x_{0} \\ u=u_{0}}}$. The equation (13) does not include higher order terms of Taylor series which depend on $\Delta u$ because the system is linear with respect to the control vector. Although it is difficult to obtain the exact value of $\left[f\left(x^{* p}, u^{* p}\right)-f\left(x^{p}, u^{p}\right)\right]$ i.e., $(L-A)$, it is possible to obtain a bound on $\|(L-A)\|$ over a range of $\left(x^{* p}, u^{* p}\right)$. This bound is used in the control design. We rewrite system (13) in terms of the block shown in Fig. 6.

Let

$$
(L-A) \Delta x=B_{2} \xi, \quad \text { and } \quad \xi=\tilde{\phi}(t) \tilde{C}_{1} \Delta x
$$

where $\xi(t)$ is the uncertainty input. Equation (14) can be written as 


$$
(L-A) \Delta x=B_{2} \tilde{\phi}(t) \tilde{C}_{1} \Delta x
$$

The nominal system is

$$
\Delta \dot{x}=A \Delta x+B_{1} \Delta u+B_{2} \xi .
$$

Matrices $B_{2}$ and $\tilde{C}_{1}$ are chosen as

$$
\begin{gathered}
B_{2}=\operatorname{diag}\left(0, \frac{X_{d}-X_{d}^{\prime}}{T_{d o}^{\prime}}, \frac{1}{2 H}, \frac{1}{T_{r}}, \frac{X_{s}-X_{s}^{\prime}}{T_{d o m}^{\prime}}, \frac{1}{2 H_{m}}, \frac{X_{s}-X_{s}^{\prime}}{T_{d o m}^{\prime}}\right) \\
\tilde{C}_{1}=\left[\begin{array}{lllllll}
1 & 0 & 0 & 0 & 0 & 0 & 0 \\
0 & 1 & 0 & 0 & 0 & 0 & 0 \\
0 & 0 & 0 & 0 & 1 & 0 & 0 \\
0 & 0 & 0 & 0 & 0 & 1 & 0
\end{array}\right]
\end{gathered}
$$

such that

$$
(L-A)=B_{2} \tilde{\phi}(t) \widetilde{C}_{1}
$$

and the expressions for obtaining $\tilde{\phi}(t)$ can be found in [13].

We define

$$
C_{1}=\sqrt{\alpha} \tilde{C}_{1}
$$

where $\alpha$ is a scaling factor which affects the magnitude of the uncertain output $\zeta$. We assume $\phi(t)=\frac{1}{\sqrt{\alpha}} \tilde{\phi}(t)$.

The value of $\alpha$ is chosen such that

$$
\|\phi(t)\|^{2} \leq 1
$$


For any value of $\delta^{*}, E_{q}^{\prime *}, \delta_{m}^{*}, E_{m}^{\prime *}$; we can conclude that

$$
\|\xi\|^{2} \leq \alpha \leq\left\|\tilde{C}_{1} \Delta x\right\|^{2}
$$

We also define $\zeta=C_{1} \Delta x$. Hence we recover the IQC (integral quadratic constraint) given in

$$
\|\xi\|^{2} \leq\|\zeta\|^{2}
$$

To facilitate control design, the power system model is written as

$$
\begin{aligned}
\Delta \dot{x}(t) & =A \Delta x(t)+B_{1} \Delta u(t)+B_{2} \xi(t)+B_{2} w(t) \\
y(t) & =C_{2} \Delta x(t)+D_{2} \xi(t)+D_{2} w(t) \\
\zeta(t) & =C_{1} \Delta x(t)
\end{aligned}
$$

where $\zeta=\left[\delta^{*} E_{q}^{* *} \delta_{m}^{*} E_{m}^{\prime^{*}}\right]^{T}$ is the uncertainty output, $\xi(t)$ is the uncertainty input, $y(t)$ is the measured output, and $w(t)$ represents a disturbance input.

Since the transducer time constant $T_{r}$ is very small, we are able to make the approximation $V_{0} \approx V_{t}$ and therefore, output matrix $C_{2}$ is defined as

$$
C_{2}=\left[\begin{array}{lllllll}
0 & 0 & 0 & 1 & 0 & 0 & 0
\end{array}\right]
$$

This new formulation presented in this is used to design the robust excitation controllers for the underlying nonlinear power systems to improve voltage stability. The performance of the PODC and RPODC with the variation of dynamic loads are discussed in the following section. 


\section{Effects of Large Dynamic Loads with PODC and RPODC}

The effects of load variation on power system stability without any controller are shown in Fig. 7. The dashed line in Fig. 7 shows the effects of constant impedance load variation and the solid line shows the effects of induction motor load variation on power system stability. It can be seen from Fig. 7 that the system retains stable operation even with a 1200 MW constant impedance load but becomes unstable for 950 MW dynamic loads.

The designed PODC is simulated on a SMIB system with constant impedance loads and dynamic loads and the robust PODC is simulated with dynamic load. The dynamic load on the system is varied from $0 \mathrm{MW}$ to $1250 \mathrm{MW}$ and the per unit (pu) damping torque provided by the PODC, through the excitation system of the generator is calculated in each variation of loads with pu changes of speed.

The dashed line and solid line in Fig. 8 show the effectiveness of RPODC with the variation of constant impedance loads and dynamic loads, respectively. Here, the effectiveness of PODC does not vary for a wide range for the power system with constant impedance loads but the effectiveness of PODC reduces a lot with the variation of dynamic loads as shown by the solid line in Fig. 8. The RPODC provides more damping as compared to the conventional PODC which is shown by the solid line with star. The effectiveness of RPODC also reduces with increasing dynamic loads but the rate of reduction is much less as compared to the conventional PODC.

\section{Conclusion}

To investigate the effects of dynamic loads on power systems stability, the linearized model of the synchronous machine and induction motor system is presented in this paper. 
Since most of the nonlinearities in the system occur due to the interconnections, therefore the effects of interconnections are also considered in the linearization process. Then by using the concept of eigenvalues and participation factors and by varying some elements of the state matrix, the direct-axis open circuit time constant of the induction motor, $T_{d o m}^{\prime}$ is found as the parameter that affects the stability of the system. The system is also sensitive to other parameters such as the exciter gain. It is shown in this paper that the critical parameter also affects the stability of large power systems.

The effectiveness of power oscillation damping controller (PODC) on an interconnected power system with the variation of dynamic load is also demonstrated in this paper. Though the conventional PODC provides better operation for power systems with constant impedance loads, but it gets worse for power systems with dynamic loads. The RPODC has better performance as compared to conventional PODC but the effectiveness of this controller also decreases with the variation of dynamic loads. So, it can be concluded that dynamic loads have significant effect on the stability of power systems. The aim of the future research is to design a controller that can provide better performance with the variation of dynamic loads.

\section{References}

[1] X. Zhao, X. Zhang, and B. He, "Study on self organized criticality of china power grid blackouts," Energy Conversion and Management, vol. 50, no. 3, pp. 658-661, March 2009.

[2] C. P. Steinmetz, "Power control and stability of electric generating stations," AIEE Trans., vol. XXXIX, Part II, pp. 1215-1287, 1920.

[3] Report, "First report of power system stability," AIEEE Trans., pp. 51-60, 1926. 
[4] F. P. Demello and C. Concordia, "Concepts of synchronous machine stability as affected by excitation control," IEEE Trans. on Power Apparatus and Systems, vol. PAS-88, no. 4, pp. 316-329, 1969.

[5] P. Kundur, Power System Stability and Control. New York: McGraw- Hill, 1994.

[6] R. A. Ramos, A. C. P. Martins, and N. G. Bretas, "An improved methodology for the design of power system damping controllers," IEEE Trans. on Power Systems, vol. 20, no. 4, pp. 1938-1945, 2005.

[7] G. Gurrala and I. Sen, "Power system stabilizers design for interconnected power systems," IEEE Trans. on Power Systems, vol. 25, no. 2, pp. 1042-1051, 2010.

[8] M. Glickman, P. OShea, and G. Ledwich, "Estimation of modal damping in power networks," IEEE Trans. on Power Systems, vol. 22, no. 3, pp. 1340-1350, 2007.

[9] A. Dysko, W. E. Leithead, and J. O’Reilly, "Enhanced power system stability by coordinated PSS design,” IEEE Trans. on Power Systems, vol. 25, no. 1, pp. 413-422, 2010. [10] Q. Lu, Y. Sun, and S. Mei, Nonlinear Control Systems and Power System Dynamics. Boston: Kluwer Academic Publishers, 2001.

[11] G. Guo, D. J. Hill, and Y. Wang, "Global transient stability and voltage regulation for power systems," IEEE Trans. on Power Systems, vol. 16, no. 4, pp. 678-688, 2001.

[12] G. Guo, D. J. Hill, and Y. Wang, "Nonlinear output stabilization control for multimachine power systems," IEEE Tans. on Circuit and Systems- Part 1, vol. 47, no. 1, pp. 46-52, 2000.

[13] M. J. Hossain, H. R. Pota, V. Ugrinovski, and R. A. Ramos, "Voltage mode stabilization in power systems with dynamic loads," Int. J. of Electric Power and Energy Systems, vol. 32, no. 8, pp. 911-920, 2010. 
[14] O. H. Abdalla, M. E. Bahgat, A. M. Serag, and M. A. El-Sharkawi, "Dynamic load modelling and aggregation in power system simulation studies," In Proc. of $12^{\text {th }}$ International Middle-East Power Systems Conference (MEPCON), 2008.

[15] W.-S. Kao, C.-T. Huang, and C.-Y. Chio, "Dynamic load modeling in power system stability studies," IEEE Tans. on Power Systems, vol. 10, no. 2, pp. 907-914, 1995.

[16] T. J. Overbye, "Effects of load modelling on analysis of power system voltage stability," Int. J. of Elec. Power and Energy Syst., vol. 16, no. 5, pp. 329-338, 1994.

[17] B. M. Nomikos and C. D. Vournas, "Investigation of induction machine contribution to power system oscillation," IEEE Tans. on Power Systems, vol. 20, no. 2, pp. 916-925, 2005.

[18] M. A. Mahmud, M. J. Hossain, and H. R. Pota, "Investigation of critical parameters for power system stability with dynamic loads," In Proc. of 2010 IEEE PES General Meeting, 2010.

[19] A. Radunskaya, R. Williamson, and R. Yinger, "A dynamic analysis of the stability of a network of induction generators," IEEE Tans. on Power Systems, vol. 23, no. 2, pp. 657-663, 2008 .

[20] D. H. Popovic, I. A. Hiskens, and D. J. Hill, "Stability analysis of induction motor networks," Int. J. of Elec. Power and Energy Syst., vol. 20, no. 7, pp. 475-487, 1998.

[21] M. J. Hossain, H. R. Pota, V. Ugrinovoski, and R. A. Ramos, "Excitation control for large disturbances in power systems with dynamic loads," In Proc. of 2009 IEEE PES General Meeting, 2009.

[22] T. V. Cutsem and C. D. Vournas, Voltage Stability of Electric Power Systems. Norwell, MA: Kluwer Academic, 1998.

[23] C. W. Taylor, Power System Voltage Stability. New York: McGraw- Hill, 1994. 
[24] H. K. Khalil, Nonlinear Systems. New York: Prentice-Hall, 1992.

\section{List of Figures}

1 Test System

2 Sensitivity of damping with respect to the induction motor direct axis rotor open circuit time constant

3 Sensitivity of damping with respect to the exciter gain

4 10-generator, 39-bus New England System

5 Excitation System with AVR and PODC

6 Robust Control Scheme

7 Effects of load variation without PODC (Solid line-Dynamic load variation, dashed lineConstant impedance load variation)

8 Effectiveness of PODC (Solid line with star-RPODC with dynamic loads, the dashed lineConventional PODC with constant impedance loads, solid line- Conventional PODC with dynamic loads) 


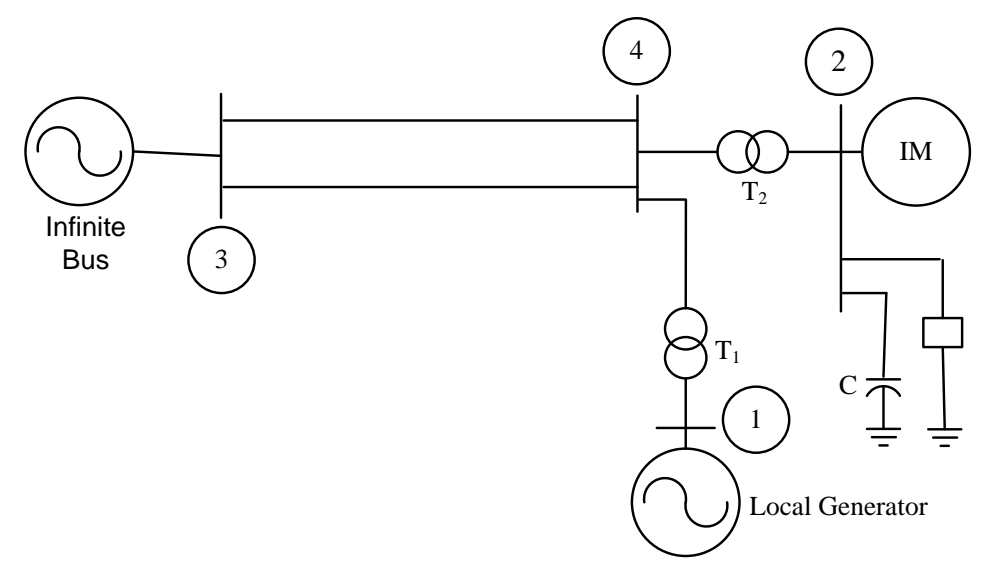

Fig. 1. Test System

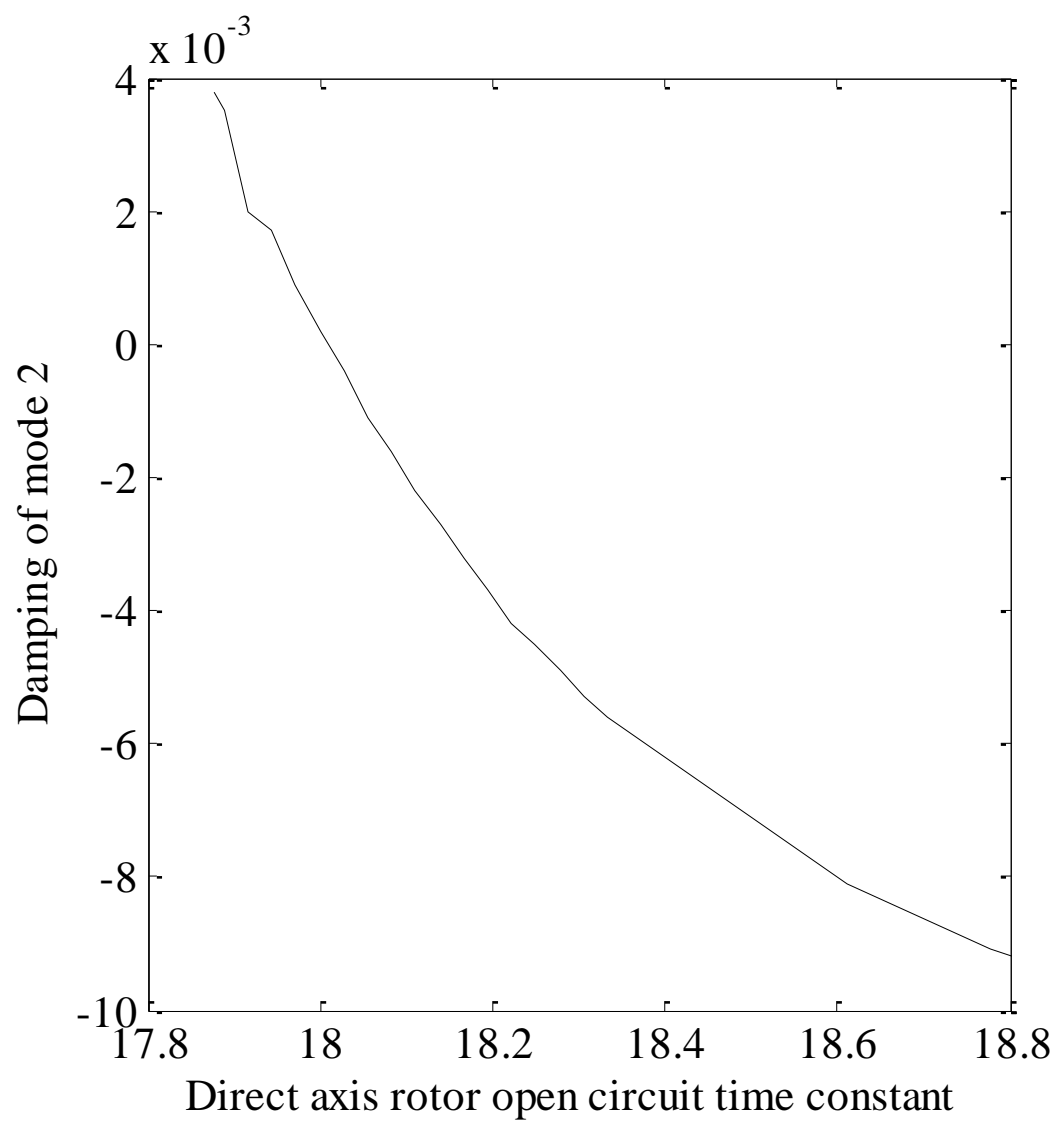

Fig. 2. Sensitivity of damping with respect to the induction motor direct axis rotor open circuit time constant 


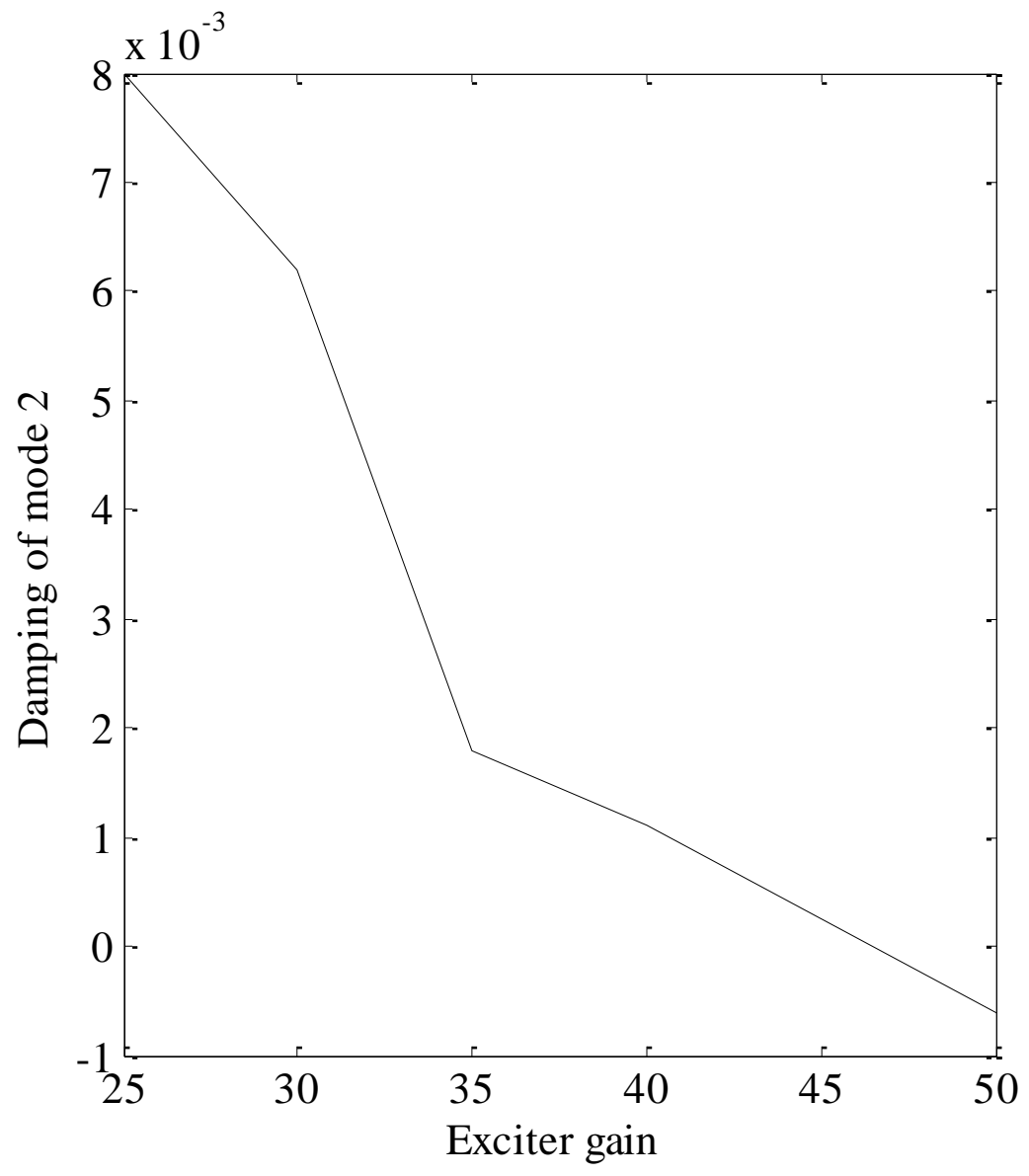

Fig. 3. Sensitivity of damping with respect to the exciter gain 


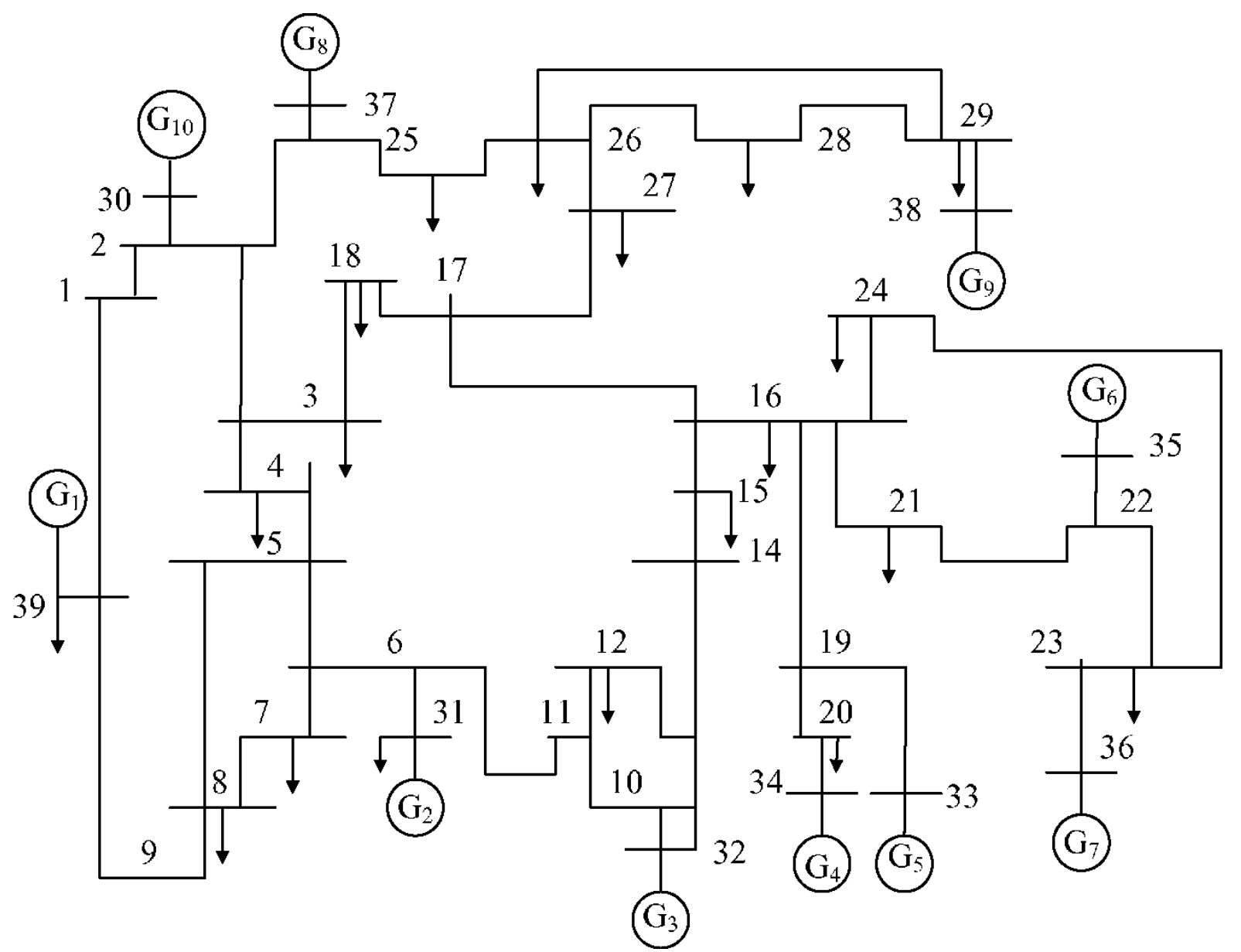

Fig. 4. 10-generator, 39-bus New England System 


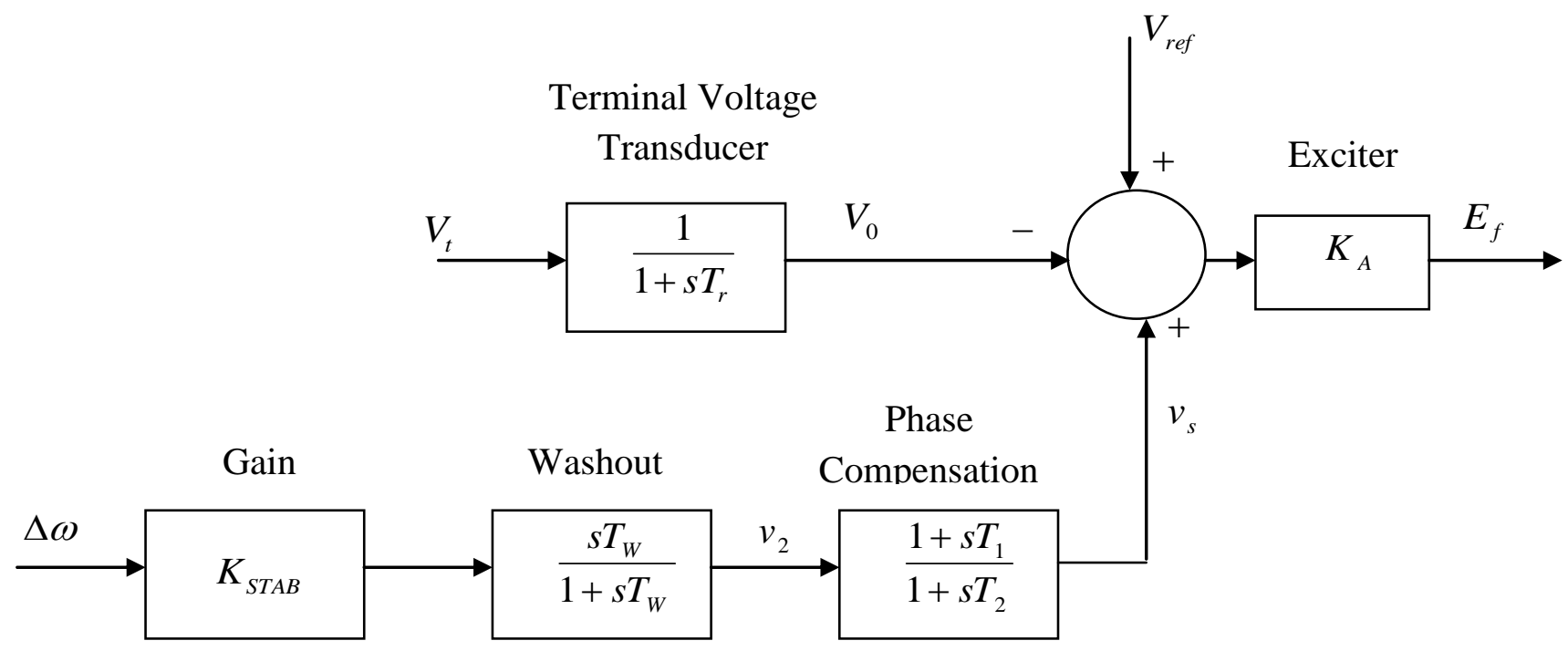

Fig. 5. Excitation System with AVR and PODC

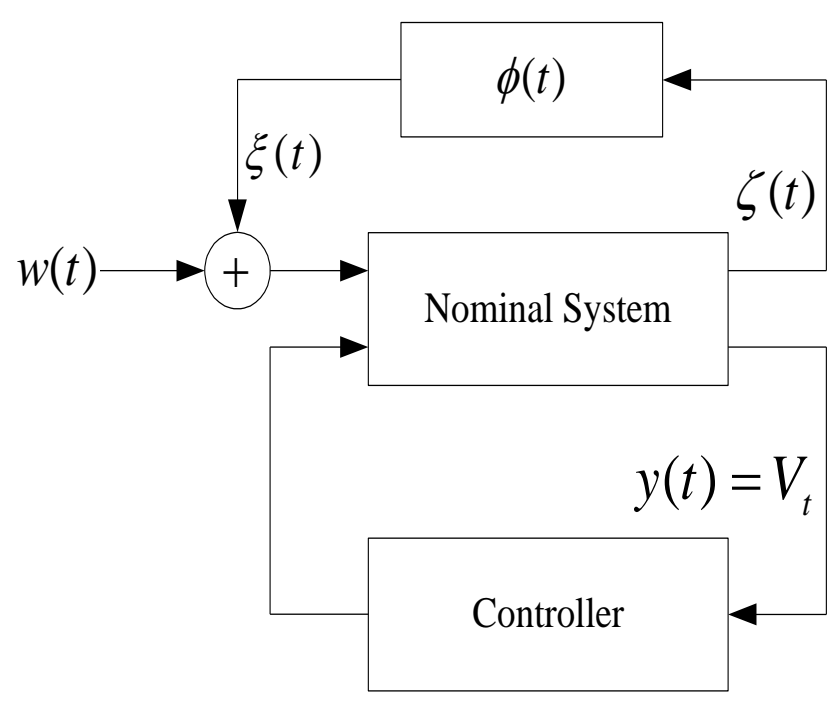

Fig.6. Robust Control Scheme 


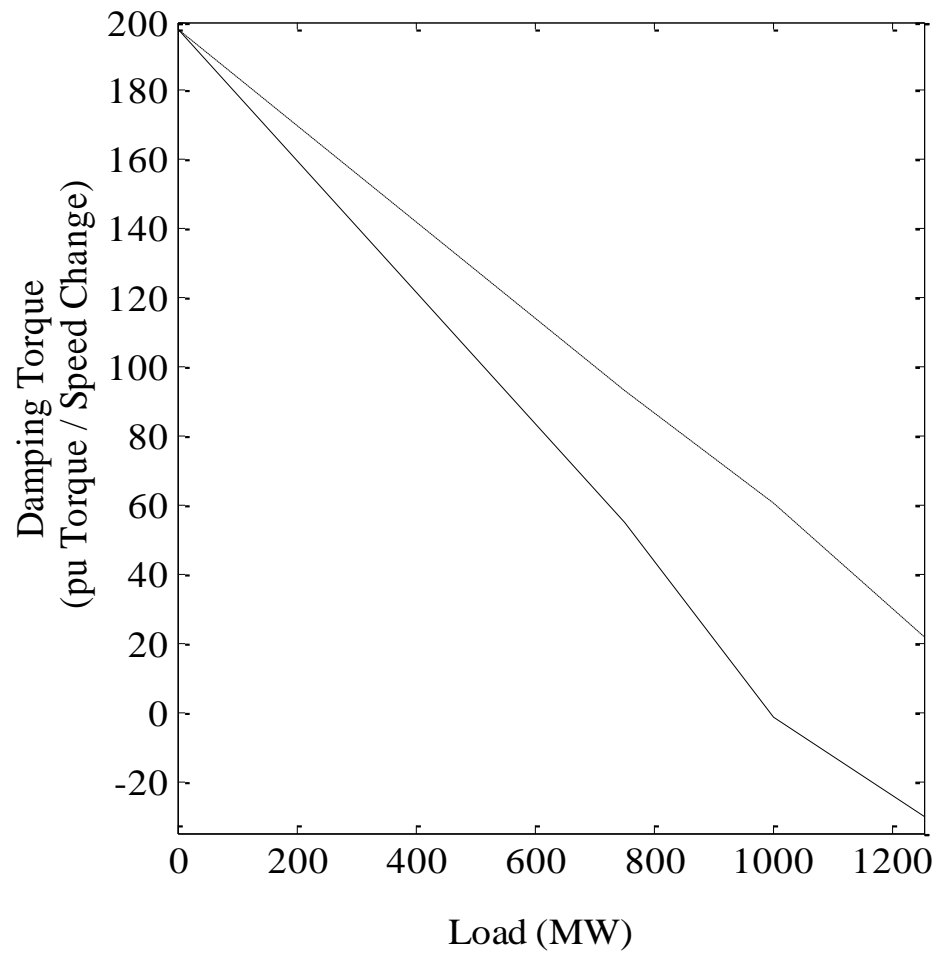

Fig. 7. Effects of load variation without PODC (Solid line-Dynamic load variation, dashed line-Constant impedance load variation)

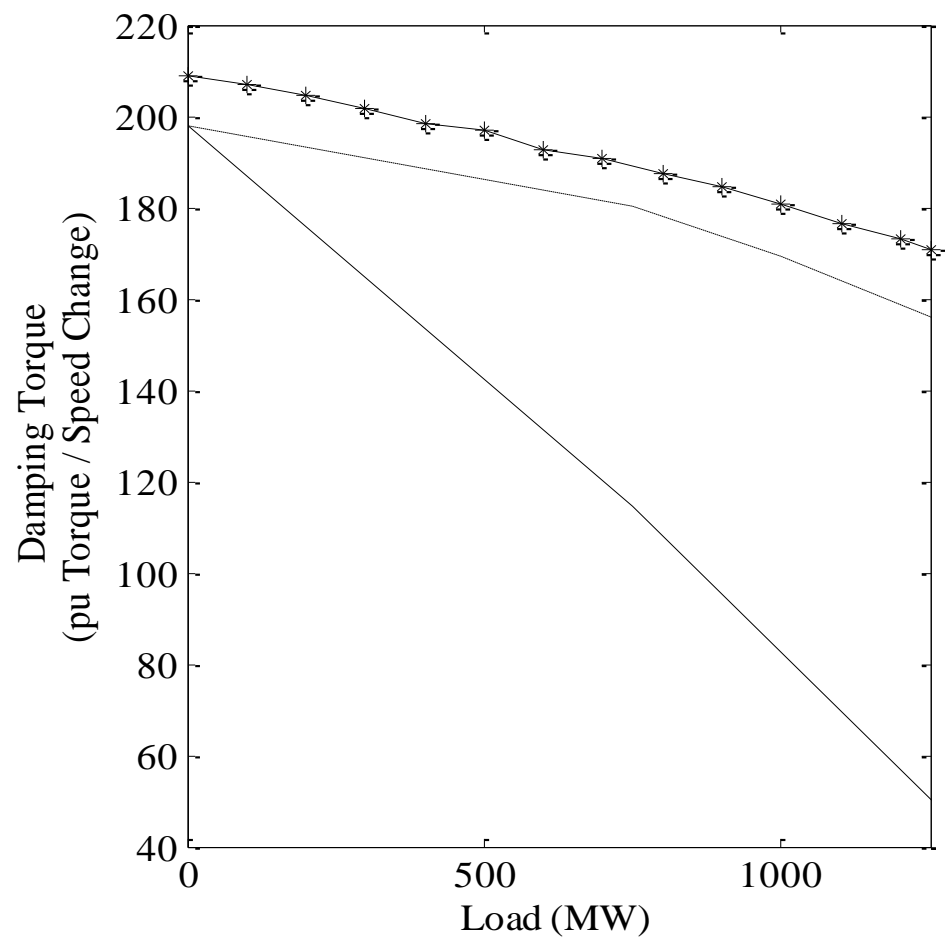

Fig. 8. Effectiveness of PODC (Solid line with star-RPODC with dynamic loads, the dashed line- Conventional PODC with constant impedance loads, solid line- Conventional PODC with dynamic loads 